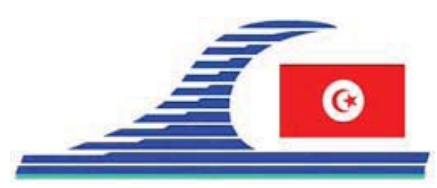

Conférence Méditerranéenne Côtière et Maritime

EDITION 1, HAMMAMET, TUNISIE (2009)

Coastal and Maritime Mediterranean Conference

Disponible en ligne - http://www.paralia.fr-Available online

\title{
Digues sous hautes sollicitations : diagnostic et réhabilitation
}

\author{
Laurent DELABY ${ }^{1}$, Claude LOUIS ${ }^{2}$
}

1. Delaby Laurent Ingénieurs Conseils, Tél : +33 (0)6 8316 8996, l.delaby@yahoo.fr

2. Claude Louis Ingénieurs Conseils, Tel : +33 (0)633306853,louiscca@msn.com

\section{Introduction}

Les digues maritimes sont des ouvrages dont la fonction est diverse : elles visent à protéger les ports et les bateaux qu'ils abritent de la houle, les bassins portuaires des sédiments, elles servent à canaliser le cours des fleuves se jetant dans la mer, elles protègent les villes des tempêtes et des inondations. Plus que toute autre construction humaine, de par leur poids, ces ouvrages sont le fruit d'un long labeur courant au travers des générations, les Hommes s'y sont souvent repris à plusieurs fois avant de parvenir à les construire, ils sont massifs, lourds et difficiles à réaliser dans un environnement dont la violence est légendaire, ces digues sont souvent soumises à l'action continue des vagues qui les érodent et leur entretien est toujours un défi à relever de manière constante et continue dans le temps et l'espace, sans répit. Dans ce contexte, cet entretien nécessite de connaître les processus d'érosion des digues, les temps et les quantités en jeux. Les investigations in situes des digues à talus permettent d'avoir une estimation de leur état intérieur, ce type d'intervention peut être délicat à réaliser car les conditions d'intervention sont susceptibles d'être périlleuses, elles apportent cependant une vue directe de l'intérieur de la digue. D'autres méthodes d'investigations telles que le photo- profil sont susceptibles d'apporter des informations sur le volume de cavités inaccessibles. Un suivi régulier des digues en maçonnerie ainsi qu'un entretien soutenu permet de prévenir que les désordres actuels ne ruinent à brève échéance les ouvrages. Nous exposons l'exemple des digues de la ville de Ault en Picardie (80) pour montrer qu'il est possible de dire que l'entretien des digues est une science qui n'en est encore qu'à ses débuts, l'entretien au coup par coup entraîne souvent des dépenses lourdes qui pourraient être réduite s'il il y avait une continuité plus grande, des méthodes d'entretien diachroniques, c'est-à-dire structurée dans le temps et la durée, peuvent être élaborées pour maintenir les ouvrages. De même de la digue d'Antifer sur laquelle nous attendons toujours l'autorisation de poursuivre nos recherches afin de pouvoir identifier les constantes de temps propres aux pathologies : la main des jeunes entreprises est tendue vers le Grand Port Maritime du Havre. Notre contribution se présente sous la forme d'une tentative d'élaboration de telles méthodes diachroniques inscrites dans la durée dont le but principal est la minimisation des coûts d'entretien des digues, pour leur entretien et leur sauvegarde. Car les enjeux humains liés à ces digues sont importants.

DOI: $10.5150 / \mathrm{cmcm} .2009 .004$ 


\section{Méthode générale d'entretien}

1.1. Une idée de base : la structuration du temps

L'idée de base de notre méthode repose sur le constat que bien souvent, la carence de constance dans le suivi des digues est le principal facteur de ruine des ouvrages. L'on pense par exemple à cette histoire apprise à la maternelle de cet enfant qui aux Pays-Bas sauva sa ville en mettant son doigt dans la petite fuite de la digue : son doigt empêcha le désordre de prendre une ampleur catastrophique. Bien souvent aussi, les digues sont construites au coup par coup, et une fois qu'elles sont construites, on les oublie les laissant à elles mêmes, c'est-à-dire que l'entretien n'est souvent pas « construit » dans le temps, il est fluctuant et variable. En reprenant des termes d'ethnologie, il existe dans la société des structures synchroniques et des structures diachroniques. Les structures synchroniques sont celles que l'on peut voir dans le présent, l'on peut alors dire que les digues sont des structures synchroniques, il est possible de voir leur existence dans le présent en allant par exemple les visiter ou les inspecter. Les structures diachroniques dont nous appelons à la construction sont dans cet esprit la méthode temporelle d'entretien, la planification des coûts d'entretien dans le temps, la mobilisation des énergies des jeunes et des jeunes entreprises, le provisionnement régulier de ces fonds d'entretien, et le suivi régulier des ouvrages assorti par des interventions d'entretien régulières. Cette méthode est économique car en maintenant l'intégrité des ouvrages elle limite les travaux d'entretien, et en répartissant les coûts dans le temps elle en rend la charge acceptable.

\subsection{Une méthode simple et efficace de diagnostic : le relevé visuel des désordres}

L'inspection des ouvrages en maçonnerie sera orientée sur :

- l'identification de la nature des parements observés; le relevé des dimensions courantes des moellons, pierres de taille et briques ainsi que l'état des joints ; l'état d'altération des pierres et les signes d'abrasion ou d'usure

- les ouvertures, les désaffleurements, les éclatements et les étendues des fissures ou fractures des parements; les déformations et basculements éventuels des ouvrages ; le relevé des zones de suintement et de défaut d'étanchéité; les affaissements ou défauts d'horizontalité des parements

L'inspection des ouvrages de type digue à talus sera orientée sur :

- l'aspect et l'état des parements et de la carapace; le relevé des dimensions courantes et de l'étendue de la structure ; les déformations, disjointoiements, désaffleurements et basculements éventuels

- les entraînements éventuels de matériaux; la mesure des cavités d'érosion par une inspection de type spéléologique

L'inspection des ouvrages ou parties d'ouvrages en béton et béton armé sera orientée sur : 
- l'aspect et l'état des parements en béton; signes d'abrasion ou d'usure et les zones de désagrégation du béton; les éraflures, épaufrures et éclats de béton

- le relevé des zones de délavage, de cavités, de fissures ou fractures, faïençage superficiel du béton; la mise à nu d'armatures ou décollement du béton d'enrobage

- les déformations, disjointoiements, désaffleurements et basculements éventuels des ouvrages ; le relevé des zones suintement et de défaut d'étanchéité; les affaissements ou défauts d'horizontalité des parements

Les désordres sont ensuite classés suivant le degré d'urgence de l'intervention :

\begin{tabular}{|c|c|c|}
\hline Indice d'Etat & Nature de l'intervention & $\begin{array}{l}\text { Degré d'urgence } \\
\text { d'intervention }\end{array}$ \\
\hline 1 & \multirow{2}{*}{$\begin{array}{c}\text { CURATIF } \\
\text { (ouvrage à problème) }\end{array}$} & Intervention immédiate \\
\hline 2 & & Court terme (1à2ans) \\
\hline 3 & \multirow{2}{*}{$\begin{array}{l}\text { PREVENTIF } \\
\text { (Entretien courant) }\end{array}$} & Moyen terme (3à4ans) \\
\hline 4 & & Faible \\
\hline
\end{tabular}

\section{Observations}

\subsection{Observations sur les digues de Ault}

Ces observations nous conduisent à proposer une méthode soutenue d'entretien qui évite d'avoir à reconstruire des digues ruinées et maintienne les digues existantes en agissant au niveau de l'atteinte superficielle des parements.

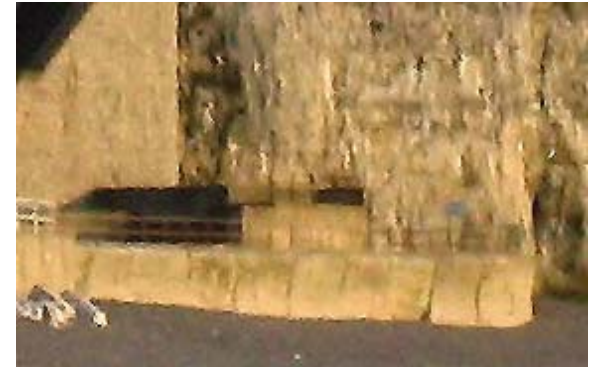

\section{Septembre 2005}

Seuls les parements sont usés. Une intervention aurait protégé la structure.

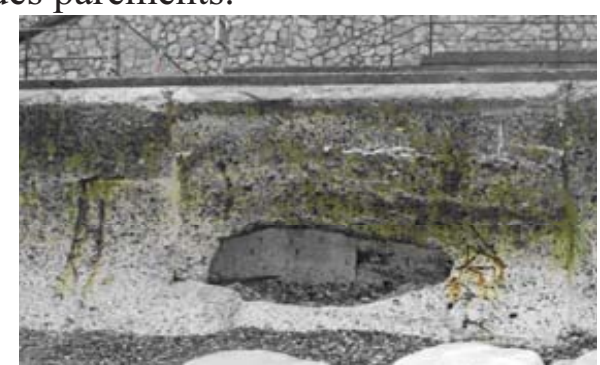

\section{Septembre 2007}

De nombreuses cavités sont apparues. La structure de l'ouvrage est atteinte.

2.2. Observations sur la digue d'Antifer-Inspection spéléologique

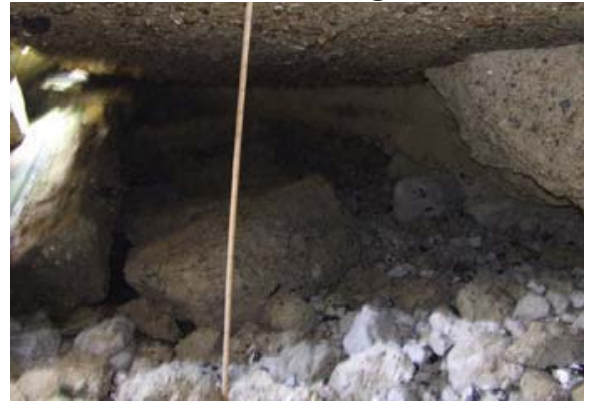

Grosse cavité d'environ $10 \mathrm{~m}$ de large, $2 \mathrm{~m}$ de hauteur et $5 \mathrm{~m}$ de profondeur.

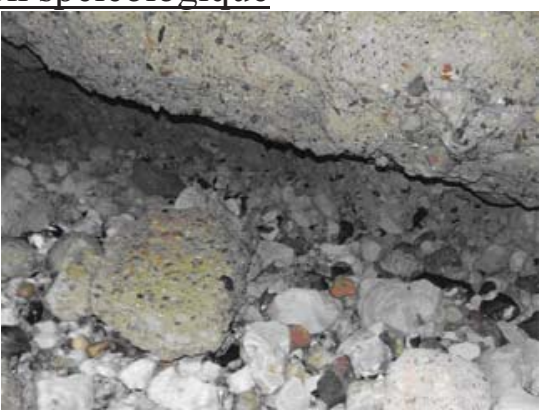

Le noyau calcaire est atteint. Le niveau du talus est très inférieur dans cette zone 


\subsection{Digue de Bayonne - inspection spéléologique}

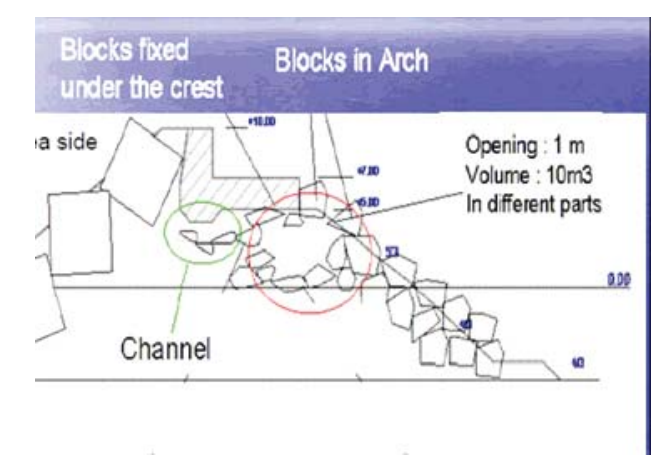

De véritables «cavernes" ont été repérées mettant en évidence une érosion interne importante.

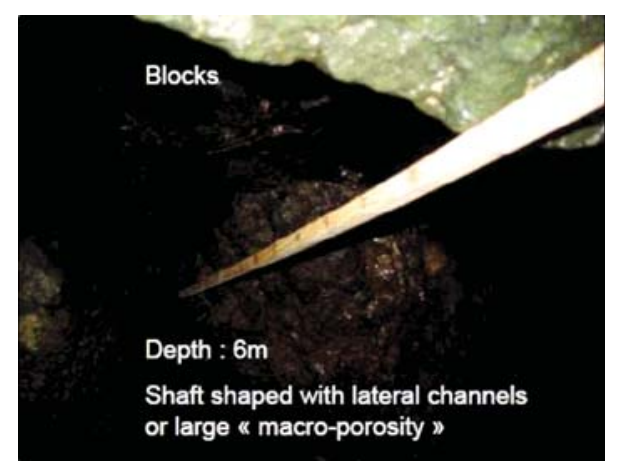

Nous avons aussi observé des cavités multiples communiquant les unes avec les autres.

\section{Préconisations}

La première préconisation consiste simplement, dans un secteur où l'on apprend la mécanique des sols et des solides, à incarner le mot " cohésion » sociale dans les faits. Le mot « cohésion » désigne selon le dictionnaire LITTRÉ (1863) : « force en vertu de laquelle les particules solides se tiennent entre elles. Dans les corps la cohérence est l'effet de la cohésion. » Aussi, l'on espère une cohésion plus grande de la société avec des liens plus forts entre les différents acteurs, notamment entre les organismes existants et les jeunes entreprises, les jeunes diplômés, pour une société plus solide. Dans le domaine de l'entretien des digues, cela conduit simplement les acteurs à avoir une propension plus grande à travailler ensemble, en particulier avec les jeunes, les jeunes entreprises, les organismes de recherche, les communes et les Ports Autonomes. Avoir confiance en les jeunes et en l'avenir. La deuxième préconisation est aussi simple que la précédente : assurer un suivi régulier des digues et un entretien structuré et continu dans le temps. La troisième préconisation va dans le sens des deux précédentes : faire l'effort de lancer des petits marchés en direction des jeunes et les jeunes entreprises, les encourager, en considérant que chaque contribution est importante, en particulier celles de ceux qui sont motivés. L'évidence conduit à ce que la cohésion des acteurs composant la société, les liens qui les unissent, leur propension à travailler ensemble, se retrouve ensuite dans la cohésion des constructions de la société, tout simplement parce que l'entretien des ouvrages ne peut être que le fruit d'une action commune.

Cet entretien maintient l'intégrité des constructions, leur pérennité, et leur solidité. Il n'y a à écrire cela que la simple cohérence.

\section{Bibliographie}

LITTRÉ E. (1863). Dictionnaire Le Littré. Deuxième édition, 1872-1877. Disponible en ligne sur : http://francois.gannaz.free.fr/Littre/accueil.php 